Series A

I. MATHEMATICA

500

\title{
SCHLICHT FUNCTIONS \\ WITH A QUASICONFORMAL EXTENSION
}

BY

OLLI LEHTO

HELSINKI 1971

S U O M A A I N N TIEDEAKATEM I

doi:10.5186/aasfm.1971.500 
Copyright (C) 1971 by

Academia Scientiarum Fennica

Communicated 10 September 1971 


\section{Schlicht functions with a quasiconformal extension}

1. Let $f$ be a schlicht analytic function in $|z|>1$ with a power series expansion $f(z)=z+a_{0}+a_{1} / z+\ldots$ A simple geometric argument shows that the coefficients satisfy the inequality $\sum n\left|a_{n}\right|^{2} \leqq 1$ (»Flächensatz»).

In this paper we introduce the additional hypothesis that $f$ possesses a quasiconformal extension to the unit disc. More exactly, $f$ is assumed to be homeomorphic in the whole plane and a generalized $L^{2}$-solution of a Beltrami differential equation $f_{\bar{z}}=\mu f_{z}$ in $|z|<1$, where $|\mu(z)| \leqq k<1$. From a representation of $f$ by means of a generalized Cauchy integral formula which takes account of the quasiconformal extension we deduce that the Flächensatz is then valid in the form $\sum n\left|a_{n}\right|^{2} \leqq k^{2}$. The estimate is sharp, equality holding if and only if $f(z)=z+a_{0}+a_{1} / z$ in $|z|>1$, $f(z)=z+a_{0}+a_{1} \bar{z}$ in $|z| \leqq 1$.

This result allows some immediate conclusions. From the special case $\left|a_{1}\right| \leqq k$ it follows that every conformal mapping $f$ of $|z|>1$ having a quasiconformal continuation with $|\mu(z)| \leqq k$ satisfies the inequality $\sup \left(|z|^{2}-1\right)^{2}\left|S_{f}(z)\right| \leqq 6 k$, where $S_{f}=\left(f^{\prime \prime} \mid f^{\prime}\right)^{\prime}-\frac{1}{2}\left(f^{\prime \prime} \mid f^{\prime}\right)^{2}$ denotes the Schwarzian derivative of $f$. From $\left|a_{1}\right| \leqq k$ we can also deduce results for functions $f$ schlicht in the unit disc and with the standard normalization $f(z)=z+a_{2} z^{2}+\ldots$ For instance, if $f$ admits a quasiconformal extension such that $|\mu(z)| \leqq k$ and $f(\infty)=\infty$, then $\left|a_{2}\right| \leqq 2 k$. These estimates are also sharp and extremal functions can be determined.

Added to the proof. After this work had already been surrendered for publication, an important paper by Reiner Kühnau appeared (Verzerrungssätze und Koeffizientenbedingungen vom Grunskyschen Typ für quasikonforme Abbildurgen, Math. Nachr. 48 (1971), containing most of the results presented here. Kühnau's main theorems are more general, and so our proofs, rather different from those of Kühnau, are perhaps more straightforward.

2. Let $f$ be a schlicht analytic function in $|z|>1$ with the development $f(z)=z+a_{0}+a_{1} / z+\ldots$, and with a quasiconformal extension to the plane. Then $f$ possesses generalized $L^{2}$-derivatives, and Pompeiu's formula 
A. I. 500

can be applied to $f$ in every finite disc. Consequently, if $z$ is a point in a disc $|\zeta|<\varrho$, then

$$
f(z)-z=\frac{1}{2 \pi i} \int_{|\zeta|=\varrho} \frac{f(\zeta)-\zeta}{\zeta-z} d \zeta-\frac{1}{\pi} \int_{|\zeta|<\varrho} \int_{\zeta} \frac{f_{\xi}(\zeta)}{\zeta-z} d \sigma_{\zeta},
$$

where $d \sigma$ denotes the euclidean element of area. Here we can let $\varrho \rightarrow \infty$, since $f(\zeta)-\zeta$ tends to the finite limit $a_{0}$ as $\zeta \rightarrow \infty$, and $f_{\xi}(\zeta)=0$ if $|\zeta|>1$. Hence,

$$
f(z)-z-a_{0}=\frac{1}{\pi} \int_{i=1} \int_{<1} \frac{f_{\xi}(\zeta)}{z-\zeta} d \sigma_{;}
$$

for every finite $z$.

Differentiation with respect to $z$ gives

$$
f_{z}(z)-1=\left(H f_{\bar{z}}\right)(z)
$$

almost everywhere, where $H$,

$$
\left(H f_{\bar{z}}\right)(z)=-\frac{1}{\pi} \int_{\zeta=1} \int_{<1} \frac{f_{\bar{\xi}}(\zeta)}{(z-\zeta)^{2}} d \sigma_{\xi},
$$

is the two-dimensional Hilbert-transformation. (For the proof of (2) and the interpretation of the singular integral in the definition of $H$ we refer to [2].)

Since the Hilbert-transformation is an isometry in $L^{2}$, we have

$$
\int_{\tilde{z}^{\prime}:<1}\left|f_{\tilde{z}}(z)\right|^{2} d \sigma=\iint\left|\left(H f_{\tilde{z}}\right)(z)\right|^{2} d \sigma,
$$

where the right-hand integral is extended over the whole plane.

3. Making use of the above formulae $(1-3)$ we can now establish our Flächensatz:

Theorem. Let $f$,

$$
f(z)=z+a_{0}+\frac{a_{1}}{z}+\frac{a_{2}}{z^{2}}+\ldots
$$

be a schlicht analytic function in $|z|>1$ with a quasiconformal extension to the plane such that $|\mu(z)| \leqq k$. Then

$$
\sum_{n=1}^{\infty} n\left|a_{n}\right|^{2} \leqq k^{2}
$$

with equality if and only if 
(6)

$$
f(z)=\left\{\begin{array}{lll}
z+a_{0}+a_{1} / z \text { for } & |z|>1 \\
z+a_{0}+a_{1} \bar{z} \text { for } & |z| \leqq 1
\end{array}\right.
$$

Proof. By the classical Flächensatz, the area of the set of values omitted by the restriction of $f$ to $|z|>1$ equals $\pi\left(1-\Sigma n\left|a_{n}\right|^{2}\right)$. Now this is the area of the image of the unit disc under the extended $f$. Hence, expressing the Jacobian of $f$ in terms of the complex derivatives, we obtain

$$
\int_{|z|} \int_{<1}\left(\left|f_{z}(z)\right|^{2}-\left|f_{\bar{z}}(z)\right|^{2}\right) d \sigma=\pi\left(1-\sum_{n=1}^{\infty} n\left|a_{n}\right|^{2}\right) .
$$

Since $f_{\bar{z}}=\mu f_{z}$ almost everywhere in $|z|<1$ and $|\mu(z)| \leqq k$, it follows that

$$
\frac{1}{\pi} \int_{|z|<1}\left|f_{\bar{z}}(z)\right|^{2} d \sigma \leqq \frac{2 k}{1-k^{2}}\left(1-\sum_{n=1}^{\infty} n\left|a_{n}\right|^{2}\right)
$$

In order to derive a lower estimate for the above integral we first conclude from (3) and (2) that

$$
\iint_{|z|<1}\left|f_{\tilde{z}}(z)\right|^{2} d \sigma=\iint\left|f_{z}(z)-1\right|^{2} d \sigma \geqq \iint_{|z|>1}\left|f^{\prime}(z)-1\right|^{2} d \sigma .
$$

Evaluating the right-hand integral in terms of the coefficients in (4) we obtain

$$
\sum_{n=1}^{\infty} n\left|a_{n}\right|^{2} \leqq \frac{1}{\pi} \int_{\tilde{z}_{1}} \int_{<1}\left|f_{\bar{z}}(z)\right|^{2} d \sigma
$$

In conjunction with (7), this implies (5).

For the function (6) we have $\mu(z)=a_{1}$ for every $z,|z|<1$, so that (5) holds as an equality.

Suppose, on the other hand, that $f$ is a function for which (5) is true as an equality. We then have equality in (8), which is only possible if $f_{z}(z)=1$ almost everywhere in $|z|<1$. In this case, $f_{\bar{z}}=\mu$ a.e., and (1) becomes

$$
f(z)=z+a_{0}+\frac{1}{\pi} \int_{\zeta \zeta \mid<1} \frac{\mu(\zeta)}{z-\zeta} d \sigma_{\zeta}
$$

Here

$$
\psi(z)=\frac{1}{\pi} \int_{|\zeta|<1} \frac{\mu(\zeta)}{z-\zeta} d \sigma_{\zeta}
$$


defines a function $\psi$ with generalized $L^{2}$-derivatives, since $f$ has such derivatives. From (10) it follows that

$$
\psi_{z}=f_{z}-1=0, \quad \psi_{\bar{z}}=f_{\bar{z}}=\mu
$$

almost everywhere in $|z|<1$. We conclude that $\bar{\psi}$ is an analytic function of $z$ in the unit disc and $\bar{\psi}^{\prime}=\bar{\mu}$ a.e. Since we must also have equality in (7), $|\mu(z)|=k$ almost everywhere. Thus $\bar{\psi}^{\prime}$, having a constant absolute value, is constant. Consequently, $\mu$ is constant in $|z|<1$, and (6) follows from (10) by direct computation.

Geometrically, (5) can be expressed as follows: $f$ maps the unit disc onto a domain with area $\geqq \pi\left(1-k^{2}\right)$. Equality holds only for the functions (6).

4. For our applications the following special case is sufficient.

Corollary 1. Under the hypotheses of the Theorem,

$$
\left|a_{1}\right| \leqq k
$$

with equality only for the functions (6).

Proof. The inequality (11) is of course a special case of (5). We remark, however, that (11) can be derived without the use of the Hilbert-transformation. In fact, multiplying both sides of (1) by $z$ and letting $z \rightarrow \infty$ we obtain

$$
a_{1}=\frac{1}{\pi} \int_{|\zeta|<1} \int_{\overline{<} 1}(\zeta) d \sigma
$$

Hence,

$$
\left|a_{1}\right|^{2} \leqq \frac{1}{\tau} \int_{|z|} \int_{<1}\left|f_{\bar{z}}(z)\right|^{2} d \sigma,
$$

which replaces the stronger inequality (9). From $(\overline{7})$ we see that

$$
\frac{1}{\pi} \int_{|z|} \int_{<1}\left|f_{\bar{z}}(z)\right|^{\prime 2} d \sigma \leqq \frac{2 k}{1-k^{2}}\left(1-\left.a_{1}\right|^{2}\right),
$$

and (11) follows.

5. It is well known that the Schwarzian derivative $S_{f}$ of a conformal mapping $f$ of $|z|>1$ satisfies the inequality $\left(|z|^{2}-1\right)^{2}\left|S_{f}(z)\right| \leqq 6$. Corollary 1 yields immediately the following generalization important in the theory of Teichmüller spaces: 
Corollary 2. Let $f$ be a schlicht analytic function in $|z|<1$ with a quasiconformal extension to the plane such that $|\mu(z)| \leqq k$. Then

$$
\left(|z|^{2}-1\right)^{2}\left|S_{f}(z)\right| \leqq 6 k
$$

for every z, $|z|>1$. Equality holds at the point $z$ if and only if $f$ is a linear transformation of $g+k / g$, where $g$ is a conformal self-mapping of the exterior of the unit disc with pole at $z$.

Proof. Given a point $z_{0},\left|z_{0}\right|>1$, let $g$ be a conformal self-mapping of $|z|>1$ with pole at $z_{0}$. By choosing a linear transformation $h$ suitably we achieve that $\phi=h \circ f \circ g^{-1}$ has a development $z+a_{1} / z+\ldots$ Considering the transformation rules for the Schwarzian derivative we have

$$
\left(|g(z)|^{2}-1\right)^{2}\left|S_{\phi}(g(z))\right|=\left(|z|^{2}-1\right)^{2}\left|S_{f}(z)\right| .
$$

Since $\phi$ has a quasiconformal extension to the plane such that the supremurn of the absolute value of the complex dilatation agrees with $\sup |\mu(z)|$, it thus follows from (13) and Corollary 1 that

$$
\left(\left|z_{0}\right|^{2}-1\right)^{2}\left|S_{f}\left(z_{0}\right)\right|=\lim _{\zeta \rightarrow \infty}\left(|\zeta|^{2}-1\right)^{2}\left|S_{\phi}(\zeta)\right|=6\left|a_{1}\right| \leqq 6 k .
$$

Equality holds in (12) if and only if $\phi(z)=z+a_{1} / z$. From this we conclude that the extremal functions are as asserted.

It follows from the continuity of the left hand expression in (12) that (12) is true at $z=\infty$ also. Equality holds if and only if $f$ is a linear transformation of $z+k e^{i \vartheta} / z$, $\vartheta$ real.

6. A trick well known in the classical theory of schlicht functions makes it possible to derive from (11) an estimate for the second coefficient of a function normalized and schlicht in the unit disc.

Corollary 3. Let $f$,

$$
f(z)=z+a_{2} z^{2}+\ldots
$$

be a schlicht analytic function in the unit disc with a quasiconformal extension to the plane such that $|\mu(z)| \leqq k$. Then

$$
\left|a_{2}+\frac{1}{f(\infty)}\right| \leqq 2 k .
$$

The bound is sharp.

Proof. The function $h$, defined by

$$
h(z)=\left(\frac{1}{f\left(1 / z^{2}\right)}-\frac{1}{f(\infty)}\right)^{1 / 2},
$$

is a schlicht analytic function in $|z|>1$ and possesses a quasiconformal 
continuation to the plane such that the supremum of the absolute value of the complex dilatation is equal to sup $|\mu(z)|$. Furthermore,

$$
h(z)=z-\frac{1}{2}\left(a_{2}+\frac{1}{f(\infty)}\right) \frac{1}{z}+\ldots
$$

Hence, (14) follows from Corollary 1.

Extremal functions can easily be determined. The term $1 / f(\infty)$ is essential, as is shown, for instance, by the function $z /(1-z)$.

If $f(\infty)=\infty$, then $\left|a_{2}\right| \leqq 2 k$. Since $(6)$ is the only extremal function in Corollary 1, we have equality here if and only if $f$ is a modified Koebe function,

$$
f(z)=\frac{z}{\left(1+k e^{i \theta} z\right)^{2}},
$$

in the unit disc, with the extension

$$
f(z)=\frac{z \bar{z}}{\left(\sqrt{\bar{z}}+k e^{i \theta} \sqrt{z}\right)^{2}}
$$

for $|z| \geqq 1$.

7. From (14) we obtain the following result: Let $f$ be a quasiconformal self-mapping of the unit disc, $|\mu(z)| \leqq k$, which keeps every boundary point fixed. Then

$$
|f(0)| \leqq 2 k
$$

This estimate follows from the observation that the function $h$,

$$
h(z)= \begin{cases}z & \text { for }|z| \leqq 1 \\ 1 / f(1 / z) & \text { for }|z|>1,\end{cases}
$$

satisfies the conditions of Corollary 3. For $h$, the coefficient $a_{2}$ is zero, and so (14) implies (15).

The estimate (15) is asymptotically correct. Teichmüller [3] has proved that

$$
\max |f(0)|=v^{-1}\left(\operatorname{arcosh} \frac{1}{k}\right),
$$

where $v(r)$ is the conformal modulus of the unit disc slit along the segment $\{x \mid 0 \leqq x \leqq r\}$. Since

$$
2 k-v^{-1}\left(\operatorname{arcosh} \frac{1}{k}\right)=O\left(k^{3}\right)
$$

as $k \rightarrow 0$, (15) gives a good estimate for small values of $k$. 
8. Throughout the above representation we assumed that the given schlicht analytic function possesses a quasiconformal extension. By a theorem of Ahlfors and Weill [1], a conformal mapping $f$ of $|z|>1$ can be continued quasiconformally to the plane whenever

$$
\sup \left(|z|^{2}-1\right)^{2}\left|S_{f}(z)\right|<2 .
$$

On the other hand, the validity of the condition

$$
\left(|z|^{2}-1\right)^{2}\left|S_{f}(z)\right|<2
$$

for every $z,|z|>1$, is no longer sufficient. A counterexample is provided by the function $f$,

$$
f(z)=\log \frac{z}{z-1}
$$

In this case,

$$
\left(|z|^{2}-1\right)^{2}\left|S_{f}(z)\right|=\frac{1}{2}\left(1+\frac{1}{z \mid}\right)^{2}\left(\frac{|z|-1}{z-1 \mid}\right)^{2}<2 .
$$

However, the image of $|z|=1$ is not a quasiconformal curve as it forms a zero angle at infinity.

On the boundary of the domain onto which $f$ maps $|z|>1$ a peak pointing inwards seems to imply a larger sup $\left(|z|^{2}-1\right)^{2}\left|S_{f}(z)\right|$ than a peak pointing outwards.

University of Helsinki and

Academy of Finland 


\section{References}

[1] Ahlfors, L. V. and Werll, G.: A uniqueness theorem for Beltrami equations. Proc. Amer. Math. Soc. 13 (1962).

[2] Lehto, O. und Virtanen, K. I.: Quasikonforme Abbildungen. - Springer-Verlag 1965.

[3] Teichmüller, O.: Ein Verschiebungssatz der quasikonformen Abbildung. - Deutsche Math. 7 (1944). 